\title{
Dynamical System Analysis of a Lassa Fever Model with Varying Socioeconomic Classes
}

\author{
Ifeanyi Sunday Onah ${ }^{1}$ and Obiora Cornelius Collins $\mathbb{D}^{2}$ \\ ${ }^{1}$ Department of Mathematics, University of Nigeria, Nsukka, Nigeria \\ ${ }^{2}$ Institute of Systems Science, Durban University of Technology, Durban 4000, South Africa \\ Correspondence should be addressed to Obiora Cornelius Collins; obiora.c.collins@gmail.com
}

Received 8 May 2020; Revised 26 August 2020; Accepted 13 September 2020; Published 25 November 2020

Academic Editor: Juan Manuel Peña

Copyright (C) 2020 Ifeanyi Sunday Onah and Obiora Cornelius Collins. This is an open access article distributed under the Creative Commons Attribution License, which permits unrestricted use, distribution, and reproduction in any medium, provided the original work is properly cited.

\begin{abstract}
Lassa fever is an animal-borne acute viral illness caused by Lassa virus. It poses a serious health challenge around the world today, especially in West African countries like Ghana, Benin, Guinea, Liberia, Mali, Sierra Leone, and Nigeria. In this work, we formulate a multiple-patch Lassa fever model, where each patch denotes a socioeconomic class (SEC). Some of the important epidemiological features such as basic reproduction number of the model were determined and analysed accordingly. We further investigated how varying SECs affect the transmission dynamics of Lassa fever. We analysed the required state at which each SEC is responsible in driving the Lassa fever disease outbreak. Sensitivity analyses were carried out to determine the importance of model parameters to the disease transmission and prevalence. We carried out numerical simulation to support our analytical results. Finally, we extend some of the results of the 2-patch model to the general $n$-patch model.
\end{abstract}

\section{Introduction}

Lassa fever is an acute virus (arenavirus) caused by Lassa virus. It is hosted by a rodent called the multimammate rat (Mastomys natalensis). Lassa fever is known to be a zoonotic disease that is primarily transmitted to humans from direct contact with infected animals. It can also be transmitted through food or household items that are exposed to the urine or faeces of infected animals. There can also be cases of secondary infection through inhalation or ingestion. Lassa fever can also be transmitted from one individual to another, when such individuals share medical equipments that are contaminated. Skin breaks in humans can be a medium through which humans can get infected with Lassa fever, and also, from dust particles through the mucous membranes referred to as aerosol transmission $[1,2]$.

Lassa fever poses a serious health challenge around the world today, especially in West African countries: Ghana, Benin, Guinea, Liberia, Mali, Sierra Leone, and Nigeria [2, 3].
There has been emergence of cases in Nigeria in recent times, and this stands as a threat to humanity and good health. According to data from the World Health Organization (WHO), between January and February 2020, Nigeria has about 172 laboratory-confirmed cases with 72 deaths in 26 out of 36 states of the country including the Federal Capital Territory. The fatality rate of Lassa fever stands at about $14.8 \%$ [4]. There is also a report from WHO that "Lassa fever is endemic in Nigeria and has its peak period during the dry seasons (i.e., between December and April of every year)." This is because the Mastomys rat is been reproduced more in the wet season (i.e., May-June) [4].

Direct and indirect exposure to Mastomys rats has been found to be the main vector of infection of Lassa fever. This direct or indirect transmission stands at a scary rate of 90 $-95 \%$ in Nigeria [4]. Indirect here is contact with food or household equipments that has been exposed to the urine or faeces of the Mastomys rat. In a more general view, the statistics of Lassa fever according to the Center for Disease 
Control and Prevention (CDC) and WHO is about 100,000 to 300,000 cases, and an approximate death records of about 5,000 annually in West Africa [3].

The human population are grouped into different social strata or category. The state of each individual depends on different factors, which range from their occupation, education, and income. This position or social strata is referred to as socioeconomic status (SES) [5]. This implies that humans can be classified into different socioeconomic classes (e.g., low SEC and high SEC). Low SEC are often characterised by lack of provisions of basic amenities, low standard of living, malnutrition, unclean environment, inadequate health facilities, low income, lack of good personal hygiene, and even lack of portable drinking water. On the other hand, high SEC is characterised by good living condition, sufficient income, quality education, secure environment, good-paying jobs, affordability of necessary health care, and relative peace. It can be seen that low socioeconomic status is synonymous to underdeveloped countries, refugee camps, wardevastated areas, and where there are cases of natural disaster. Notwithstanding that all humans are susceptible to Lassa fever, the humans in low SEC are most likely to be infected with the disease considering their environment [6]. Based on this, the transmission dynamics of Lassa fever will vary across SECs, since each society is made up of different socioeconomic classes. The different SECs and how they are infected by Lassa fever will be discussed in this study. This will be achieved by formulating and analysing an appropriate mathematical epidemiological model of Lassa fever disease dynamics that incorporates different SECs. A mathematical epidemiological model for Lassa fever incorporating socioeconomic classes is geared towards improving the understanding of the transmission dynamics of the disease. With appropriate formulation of mathematical epidemiological Lassa fever model with SECs, one will have a better understanding of the effective control/intervention strategies to combat the disease with.

A number of mathematical models such as [7-14] have explored the dynamics of Lassa fever. Lassa fever has been at the center of infectious disease. Many researches are recently carried out works to expose further its transmission dynamics and presenting different approaches to its control. We present some of these new studies and their methodologies and results. Musa et al. [15] carried out a detailed study of Lassa fever epidemics in Nigeria, between 2016 and 2019. They employed the use of mathematical model in carrying out this study. Mariem et al. [16] also carried out a study on Lassa fever. Their interest was in the control of rodents as a way of eradicating Lassa fever. They also considered experimental field data. Akhmetzhanov et al. [17] studied the seasonal drivers of transmission for Lassa fever in Nigeria. By developing a mathematical model together with the data sets of human infection, they analyse the population dynamics of rodents and investigate the impact of climate variations in Lassa fever transmission. Zhao et al. [18] in their study of Lassa fever used different mathematical models such as the Richards, threeparameter logistic, Gompertz, and Weibull growth models to analyse the epidemiological features of Lassa fever epi- demics in Nigerian. In particular, they investigate how these epidemiological features differ across the regions of Nigeria at different time periods by relating the basic reproduction number with rainfall across the regions. Iacono et al. [13] use innovative modelling together with published data to estimate person-to-person contribution in Lassa fever transmission. They discovered that almost $20 \%$ of secondary cases of Lassa fever come from person-to-person transmission. Detailed analysis of Nigeria's Lassa fever incidence case was carried out by Ilori et al. [19]. They documented both the epidemiological and clinical report of Lassa fever in Nigeria and used their model to analyse Lassa fever disease dynamics in Nigeria. There are other researches carried out on Lassa fever outbreak in Nigeria. One of such is the one carried out by Roberts [20]. Also, Ajayi et al. [21] did an interesting work by carrying out expository research work on Lassa fever in Nigeria. They also used clinical epidemiological and laboratory data in analysing the impact of this disease in Nigeria.

Currently, there is no vaccine for Lassa fever and even the treatment is not $100 \%$ sure. This led Warner et al. [22] to conduct a research that looked at the vaccines that had worked in animals and its possible trials in humans. Some research work geared towards finding a vaccine for Lassa fever are reported in [23-25]. From the reviewed works above, it is evident that there is no vaccine yet and even the cure is not very effective and efficient. Therefore, other control measures should be employed in eradicating or reducing the prevalence of this disease especially in the countries where the disease is endemic. These control measures could be adequate environmental cleanliness, healthy living practices, effective and efficient rodent control, improved health system, and maintaining a good lifestyle in general $[1,2]$.

These works reviewed above have been of immense importance to understanding the dynamics of and control measures for Lassa fever disease. From the reviewed literature and to the best of our knowledge, none of these works considered socioeconomic classes (SECs) on the transmission dynamics of Lassa fever disease.

The aim of this work is to formulate a more general mathematical epidemiological model that incorporates different socioeconomic classes. The analyses of this model will contribute to existing knowledge of Lassa fever transmission dynamics so as to pave way for improved prevention mechanism for this disease.

\section{Formulation of Model Equation}

Let $N$ be the total human population susceptible to Lassa fever disease infections. This total human population $(N)$ is divided into $n$ subpopulations denoted by $N_{j}$ such that each subpopulations represent a socioeconomic class (SEC). Each SEC is subdivided into susceptible $\left(S_{j}\right)$, infected $\left(I_{j}\right)$, and recovered individuals $\left(R_{j}\right)$. The rodent population is made up of the susceptible rodents $(P)$ and the infected rodents $(Q)$. We took into consideration that the transmission can be from one individual to another or from the rodent to an 
individual when they come into contact. This is because research has proven that Lassa fever from the Mastomys rat is the most prevalent, not forgetting the fact that there is also human to human transmission $[12,18]$. Susceptible humans $S_{j}$ become infected through contact with infected humans $I_{j}$ at a rate $\beta_{j}$ and also through contact with infected rodents $Q$ at a rate $\alpha_{j}$. Infected humans $I_{j}$ recover at a rate $\rho_{j}$. Susceptible rodents $P$ become infected with Lassa virus as a result of contact with another infected rodent $Q$ at a rate $\varphi$. Next, we assume that natural birth/death occurs in SEC $j$ at a rate $\mu_{j}$. Also, natural birth/death occurs in rodents at a rate $\xi$. The assumption of equal birth and death rates (i.e., constant population) for each SEC and rats population is considered here, because we are studying the dynamics of the system for a short period of time. In the future, we will improve on this assumption so that we can study the dynamics of the disease over a long period of time.

The characteristics of both lower and higher SECs have been earlier highlighted, and this will form a basis for our choice of parameter values. The humans in the higher social strata are exposed to better sanitation, better health care practices, better living environment, and even healthier agricultural practices than humans in the lower social strata. In our model, we assume that individuals move from the lower SECs to the next higher SECs and vice versa. Therefore, the contact rates will also decrease accordingly, that is, contact rate at lower class will be higher than that of higher class. Similarly, the recovery rate of the lower class will be lower than that of the higher class. The order of arrangement of these classes is that SEC 1 is the least class and SEC $n$ is the topmost class, so SEC 2 is of a higher class than SEC 1 and SEC 3 is of a higher class than SEC 2 in that order. Based on these, we can write the inequalities connecting these classes as $\beta_{1}>\beta_{2}>\cdots>\beta_{n}, \alpha_{1}>\alpha_{2}>\cdots>\alpha_{n}$ and $\rho_{1}<\rho_{2}<\cdots<$ $\rho_{n}$. Note that individuals can migrate from lower to higher SEC as they gain more level of education, get better education, or get jobs that pay higher than their former. Also, humans in a higher SEC can suffer loss of jobs or fall into severe economic hardship, thereby leaving them with less income. This situation can make people move from a higher SEC to a lower SEC. As a result of these, we assume that $S_{j}(t)$ moves to $S_{k}(t)$ at a rate $\delta_{j k}$. Specifically, we assume that $S_{j}(t)$ can only move to the next lower SEC $S_{j-1}(t)$ or to the next higher SEC $S_{j+1}(t)$. We excluded jumping in the system to ensure smooth migration and reduce ambiguity. Furthermore, infected individuals can move from lower social strata to higher SEC as they get better health facilities or are better taken care of. On the other hand, some infected humans who have lost their source of income may move down to the lower SEC. As a result of these also, we say that $I_{j}(t)$ move to $I_{k}(t)$ at a rate $l_{j k}$ and take it that $I_{j}(t)$ can move to the next lower $\operatorname{SEC} I_{j-1}$ or to the next higher SEC $I_{j+1}$.

We do not consider movements between the recovered classes in our model, because it is assumed that the recovered class does not have a significant influence on the transmission of Lassa fever disease in the population. Given these factors, we developed the model below:

$$
\begin{aligned}
\dot{S}_{1}= & \mu_{1} N_{1}(t)-\beta_{1} S_{1}(t) I_{1}(t)-\alpha_{1} S_{1}(t) Q(t)-\mu_{1} S_{1}(t) \\
& -\delta_{12} S_{1}(t)+\delta_{21} S_{2}(t), \\
\dot{I}_{1}= & \beta_{1} S_{1}(t) I_{1}(t)+\alpha_{1} S_{1}(t) Q(t)-\left(\mu_{1}+\rho_{1}\right) I_{1}(t) \\
& -l_{12} I_{1}(t)+l_{21} I_{2}(t), \\
\dot{R}_{1}= & \rho_{1} I_{1}(t)-\mu_{1} R_{1}(t), \\
\dot{S}_{2}= & \mu_{2} N_{2}(t)-\beta_{2} S_{2}(t) I_{2}(t)-\alpha_{2} S_{2}(t) Q(t)-\mu_{2} S_{2}(t) \\
& -\delta_{21} S_{2}(t)+\delta_{12} S_{1}(t), \\
\dot{I}_{2}= & \beta_{2} S_{2}(t) I_{2}(t)+\alpha_{2} S_{2}(t) Q(t)-\left(\mu_{2}+\rho_{2}\right) I_{2}(t) \\
& -l_{21} I_{2}(t)+l_{12} I_{1}(t), \\
\dot{R}_{2}= & \rho_{2} I_{2}(t)-\mu_{2} R_{2}(t), \\
\vdots= & \vdots, \\
\dot{S}_{n}= & \mu_{2} N_{n}(t)-\beta_{n} S_{n}(t) I_{n}(t)-\alpha_{n} S_{n}(t) Q(t)-\mu_{n} S_{n}(t) \\
& -\sum_{j=n-1}^{n} \delta_{n j} S_{n}(t)+\sum_{j=n-1}^{n} \delta_{n j} S_{j}(t), \\
\dot{R}_{n}= & \rho_{n} I_{n}(t)-\mu_{n} R_{n}(t), \\
\dot{P}= & \xi Z(t)-\phi P(t) Q(t)-\xi P(t), \\
\dot{I}_{n}= & \beta_{n} S_{n}(t) I_{n}(t)+\alpha_{n} S_{n}(t) Q(t)-\left(\mu_{n}+\rho_{n}\right) I_{n}(t) \\
& \cdot-\sum_{j=n-1}^{n} l_{n j} I_{n}(t)+\sum_{j=n-1}^{n} l_{n j} I_{n}(t), \\
& \phi(t)-\xi Q(t), \\
&
\end{aligned}
$$

where $j=1,2, \cdots, n$ and $\delta_{j j}=\delta_{k k}=0$ and also $l_{j j}=l_{k k}=0 \forall j, k$. The variables of the model and their meaning are presented in Table 1. All the parameters of the model are assumed to be positive as outlined in Table 2 .

The initial conditions are assumed as follows:

$$
S_{j}(0)>0, I_{j}(0) \geq 0, R_{j}(0) \geq 0, P(0)>0, Q(0) \geq 0 .
$$

From our earlier inequalities where $\beta_{1}>\beta_{2}>\cdots>\beta_{n}, \alpha_{1}$ $>\alpha_{2}>\cdots>\alpha_{n}$ and $\rho_{1}<\rho_{2}<\cdots<\rho_{n}$, we can infer that movement from $j$ th SEC to a higher $(j+1)$ th SEC decreases contact rate $\beta_{j}$ and $\alpha_{j}$ by factors $m<1$ and $\sigma<1$, respectively, and also increases the recovery rate $\rho_{j}$ by a factor $a>1$. Thus, we can write $\beta_{j}, \alpha_{j}$, and $\rho_{j}$ as follows:

$$
\beta_{j}=m^{j-1} \beta_{1}, \quad \alpha_{j}=\sigma^{j-1} \alpha_{1}, \quad \rho_{j}=a^{j-1} \rho_{1},
$$

where $0<m, \sigma<1$, and $a>1$. 


\section{Model with Two Socioeconomic Classes}

In this section, we consider a situation where the population is made up of two SECs (i.e., lower SEC and higher SEC). The analysis of the model with just two socioeconomic classes will give better insight of the dynamics of the general $n$-SECs model (1). Setting $n=2$ in model (1), we obtain

$$
\begin{aligned}
\dot{S}_{1}= & \mu_{1} N_{1}(t)-\beta_{1} S_{1}(t) I_{1}(t)-\alpha_{1} S_{1}(t) Q(t)-\mu_{1} S_{1}(t) \\
& -\delta_{12} S_{1}(t)+\delta_{21} S_{2}(t), \\
\dot{I}_{1}= & \beta_{1} S_{1}(t) I_{1}(t)+\alpha_{1} S_{1}(t) Q(t)-\left(\mu_{1}+\rho_{1}\right) I_{1}(t) \\
& -l_{12} I_{1}(t)+l_{21} I_{2}(t), \\
\dot{R}_{1}= & \rho_{1} I_{1}(t)-\mu_{1} R_{1}(t), \\
\dot{S}_{2}= & \mu_{2} N_{2}(t)-\beta_{2} S_{2}(t) I_{2}(t)-\alpha_{2} S_{2}(t) Q(t)-\mu_{2} S_{2}(t) \\
& -\delta_{21} S_{2}(t)+\delta_{12} S_{1}(t), \\
\dot{I}_{2}= & \beta_{2} S_{2}(t) I_{2}(t)+\alpha_{2} S_{2}(t) Q(t)-\left(\mu_{2}+\rho_{2}\right) I_{2}(t) \\
& -l_{21} I_{2}(t)+l_{12} I_{1}(t), \\
\dot{R}_{2}= & \rho_{2} I_{2}(t)-\mu_{2} R_{2}(t), \\
\dot{P}= & \xi Z(t)-\phi P(t) Q(t)-\xi P(t), \\
\dot{Q}= & \phi P(t) Q(t)-\xi Q(t) .
\end{aligned}
$$

The subscript 1 in model (4) above represents the lower SEC (i.e., SEC 1), while the subscript 2 stands for the higher SEC (i.e., SEC 2).

3.1. The Basic Reproduction Number. Model (19) has a disease-free equilibrium (DFE) given as

$$
\left(S_{1}^{0}, I_{1}^{0}, S_{2}^{0}, I_{2}^{0}, P^{0}, Q^{0}\right)=\left(\frac{\delta_{21} N}{\delta_{21}+\delta_{12}}, 0, \frac{\delta_{12} N}{\delta_{21}+\delta_{12}}, 0, Z, 0\right)
$$

The DFE (5) was obtained using the fact that $S_{1}+S_{2}=N$ and at DFE, $S_{1}^{0}=N_{1}, S_{2}^{0}=N_{2}$ and solving model (4) simultaneously under these conditions. The population of susceptible in each SEC at DFE depends on the movement rates from one SEC to another. For instance, when there is equal movement rate across the SECs (i.e., $\delta_{21}=\delta_{12}$ ), then the two SECs have equal susceptible population at DFE (i.e., $S_{1}^{0}$ $=S_{2}^{0}$ ). Similarly, if more individuals moves into SEC 1 (i.e., $\delta_{21}>\delta_{12}$ ), then SEC 1 dominates at DFE (i.e., $S_{1}^{0}>S_{2}^{0}$ ). On the other hand, if more people moves into SEC 2 (i.e., $\delta_{21}<$ $\delta_{12}$ ), then SEC 2 dominates at DFE (i.e., $S_{1}^{0}<S_{2}^{0}$ ). The above results demonstrates the importance of migration rates at DFE. In the subsequent sections, we will explore the significance of migration rates in the dynamics of Lassa fever disease on a multiple socioeconomic population.
TABLE 1: Definition of variables in model (1).

\begin{tabular}{cc}
\hline$N_{j}(t)$ & Total human population in the $j t h$ SEC \\
$S_{j}(t)$ & Susceptible humans in the $j t h$ SEC \\
$I_{j}(t)$ & Infected humans in the $j t h$ SEC \\
$R_{j}(t)$ & Recovered humans in the $j t h$ SEC \\
$Z(t)$ & Total rodent population \\
$P(t)$ & Susceptible rodents \\
$Q(t)$ & Infected rodents \\
\hline
\end{tabular}

TABLE 2: Definition of parameters used in model (1).

\begin{tabular}{lr}
\hline$\beta_{j}$ & The rate of contact between $S_{j}(t)$ and $I_{j}(t)$ \\
$\alpha_{j}$ & Contact rate between $S_{j}(t)$ and $Q(t)$ \\
$\delta j k$ & Rate at which humans migrate from $S_{j}(t)$ and $S_{k}(t)$ \\
$l j k$ & Rate at which humans migrate from $I_{j}(t)$ and $I_{k}(t)$ \\
$\rho_{j}$ & Recovery rate of $I_{j}(t)$ \\
$\varphi$ & Contact rate between $P(t)$ and $Q(t)$ \\
$\mu_{j}$ & Natural birth/death rate of humans in the jth SEC \\
$\xi$ & Natural birth/death rate of rodents \\
\hline
\end{tabular}

The basic reproduction number can be understood as the average number of new infections of Lassa fever that will be seen if an infected human is brought into contact with a given population that is susceptible to Lassa fever. The basic reproduction number of model (4) is computed using the next generation matrix approach of Van Driessche and Watmough [26]. The associated next generation matrices of model (4) is

$$
F V^{-1}=\left(\begin{array}{ccc}
a_{11} & a_{12} & a_{13} \\
a_{21} & a_{22} & a_{23} \\
0 & 0 & a_{33}
\end{array}\right)
$$

where

$$
\begin{aligned}
F & =\left(\begin{array}{ccc}
\beta_{1} S_{1}^{0} & 0 & \alpha_{1} S_{1}^{0} \\
0 & \beta_{2} S_{2}^{0} & \alpha_{1} S_{2}^{0} \\
0 & 0 & \xi P^{0}
\end{array}\right), \\
V & =\left(\begin{array}{ccc}
\left(\mu_{1}+\rho_{1}\right)+l_{12} & -l_{21} & 0 \\
-l_{12} & \left(\mu_{2}+\rho_{2}\right)+l_{21} & 0 \\
0 & 0 & \xi
\end{array}\right),
\end{aligned}
$$

$$
a_{11}=\beta_{1} S_{1}^{0} b_{2} / k_{1} k_{2}+k_{1} l_{21}+k_{2} l_{12}, a_{12}=\beta_{1} S_{1}^{0} l_{21} / k_{1} k_{2}+k_{1}
$$
$l_{21}+k_{2} l_{12}, a_{13}=\alpha_{1} S_{1}^{0} / \xi, a_{21}=\beta_{2} S_{2}^{0} l_{12} / k_{1} k_{2}+k_{1} l_{21}+k_{2} l_{12}, a_{22}$ $=\beta_{2} S_{2}^{0} b_{1} / k_{1} k_{2}+k_{1} l_{21}+k_{2} l_{12}, a_{23}=\alpha_{2} S_{2}^{0} / \xi, a_{33}=\phi P^{0} / \xi, b_{1}=$ $k_{1}+l_{12}, b_{2}=k_{2}+l_{21}, k_{1}=\mu_{1}+\rho_{1}, k_{2}=\mu_{2}+\rho_{2}$. The two 
possible dominant positive real eigenvalues of the matrix $F V^{-1}$ are

$$
\lambda=\mathscr{R}_{h}, \lambda=\mathscr{R}_{r},
$$

where $\mathscr{R}_{h}=\left(\left(a_{11}+a_{22}\right)+\sqrt{\left(a_{11}+a_{22}\right)^{2}+4\left(a_{12} a_{21}-a_{11} a_{22}\right)}\right)$ 12 and $\mathscr{R}_{r}=\phi Z / \xi$. Clearly, $\mathscr{R}_{r}>0$. To show that $\mathscr{R}_{h}>0$, we need to show that $\left(a_{11}+a_{22}\right)^{2}+4\left(a_{12} a_{21}-a_{11} a_{22}\right) \geq 0$; otherwise, we obtain a complex eigenvalue. Unfortunately, to show analytically that the inequality $\left(\left(a_{11}+a_{22}\right)^{2}+4\left(a_{12} a_{21}-a_{11}\right.\right.$ $\left.a_{22}\right) \geq 0$ ) holds in general is difficult due to many different parameter combinations in the equation. However, we discovered that for various parameter values or assumptions, the inequality holds. For instance, if $l_{12}=l_{21}=0$, the inequality reduces to $\left(a_{11}-a_{22}\right)^{2}$ which is positive. Thus, the basic reproduction number $\mathscr{R}_{0}$ is the dominant positive real eigenvalue of the matrix $F V^{-1}$ and is given by

$$
\mathscr{R}_{0}=\max \left\{\mathscr{R}_{h}, \mathscr{R}_{r}\right\},
$$

provided $\left(a_{11}+a_{22}\right)^{2}+4\left(a_{12} a_{21}-a_{11} a_{22}\right) \geq 0$. Epidemiologically, $\mathscr{R}_{h}$ is the basic reproduction number attributed to humans, while $\mathscr{R}_{r}$ is the basic reproduction number related to rodents.

Next, we analyse the basic reproduction number for human population only. If we consider only the human population, its basic reproduction number according to equation (8) is

$$
\mathscr{R}_{h}=\frac{\left(a_{11}+a_{22}\right)+\sqrt{\left(a_{11}+a_{22}\right)^{2}+4\left(a_{12} a_{21}-a_{11} a_{22}\right)}}{2} .
$$

Note that $l_{12}=l_{21}=0 \Leftrightarrow a_{12}=a_{21}=0$. This implies that there is no movement of infected humans from one SEC to another. This also means that humans in one socioeconomic class will not contribute to infections in the other SEC. When this happens, $\mathscr{R}_{h}$ reduces to [27]

$$
\mathscr{R}_{h}=\max \left\{a_{11}, a_{22}\right\},
$$

where $a_{11}$ and $a_{22}$ simplifies to $a_{11}=\beta_{1} S_{1}^{0} / k_{1}$ and $a_{22}=\beta_{2} S_{2}^{0} /$ $k_{2}$ when $\left(l_{12}=l_{21}=0\right)$. Note that $a_{11}$ is the basic reproduction number associated with SEC 1, while $a_{22}$ is the basic reproduction number associated with SEC 2 . Consequently, equation (29) shows that when there is no movement of infected individuals across the SEC $\left(l_{12}=l_{21}=0\right)$, the basic reproduction number associated with humans depends on either of the SEC that dominates. So, $R_{h}=a_{11}$ if SEC 1 dominates, while $\mathscr{R}_{h}=a_{22}$ if SEC 2 dominates. Also, from (29), we can see that for $R_{h}$ to be less than unity, we must have $a_{11}<1$ and $a_{22}<1$. In a situation where either $a_{11}$ or $a_{22}$ is greater than unity, then $\mathscr{R}_{h}$ will also be greater than 1 . Therefore, in order to prevent an outbreak of Lassa fever disease in the human population, we must ensure that $a_{11}<1$ and $a_{22}<1$.
3.2. Type Reproduction Number. In this study, we are considering a heterogeneous human population where there are multiple host types. It is of importance to investigate the type reproduction number which is vital in determining the number of secondary infection that is expected in each distinct subpopulation $[28,29]$. The type reproduction number can be computed using the formula below:

$$
T=e^{1} K(I-(I-P) K)^{-1} e,
$$

where $I$ is the identity matrix, $e$ is the first unit vector and $P$ is the projection matrix on type 1 (i.e., $p_{11}=1$, and $p_{i j}=0$ for all other entries) $[28,29]$. The type reproduction number in relation with SEC 1 is

$$
T_{1}=a_{11}+\frac{a_{12} a_{21}}{1-a_{22}}
$$

Similarly, the type reproduction number associated with SEC 2 is

$$
T_{2}=a_{22}+\frac{a_{12} a_{21}}{1-a_{11}}
$$

From the equations (13) and (14), we observe that if there is no movement of infected individuals across the SECs $\left(l_{12}=l_{21}=0\right)$, then $T_{1}=a_{11}$ and $T_{2}=a_{22}$. This agrees with our earlier results on the analysis of basic reproduction numbers above. Epidemiologically, to eliminate the disease from SEC 1, we must keep $T_{1}$ below unity. Similarly, to eliminate the disease from SEC 2, we must keep $T_{2}$ below unity.

3.3. The Dominant SEC of the 2-SEC Model. Lassa fever disease has short incubation period of about 6-21 days [30]. In this sense, it seems that an infected human $I_{j}(t)$ will consider getting well first before considering improving his or her social standard like getting new jobs, education, or other sources of income. In a case where infected individuals do not move from one SEC to another SEC, (i.e., $l_{12}=l_{21}=0$ ), from equation (11), we have that the basic reproduction number for the entire human population comprising of the two SECs can be written as

$$
\mathscr{R}_{h}=\max \left\{\mathscr{R}_{h 1}, \mathscr{R}_{h 2}\right\},
$$

where $\mathscr{R}_{h 1}=\beta_{1} \delta_{21} N /\left(\mu_{1}+\rho_{1}\right)\left(\delta_{21}+\delta_{12}\right)$, and $\mathscr{R}_{h 2}=\beta_{2} \delta_{12} N /$ $\left(\mu_{2}+\rho_{2}\right)\left(\delta_{21}+\delta_{12}\right)=m \beta_{1} \delta_{12} N /\left(\mu_{2}+a \rho_{1}\right)\left(\delta_{21}+\delta_{12}\right)$. Biologically, $\mathscr{R}_{h 1}$ and $\mathscr{R}_{h 2}$ are the basic reproduction numbers associated with SEC 1 and SEC 2 , respectively [28, 29, 31]. Since $\mathscr{R}_{h}=\max \left\{\mathscr{R}_{h 1}, \mathscr{R}_{h 2}\right\}$, it tells us that either SEC 1 or SEC 2 drives Lassa fever disease outbreak in the human population. Therefore, investigating which of the SEC is driving the Lassa fever disease outbreak is crucial. This will enable us to make efficient plans in controlling the outbreak from the entire population. To investigate this, we identified the relationship that exists between $\mathscr{R}_{h 1}$ and $\mathscr{R}_{h 2}$ considering the following cases: 
(i) $\delta_{21}=\delta_{12}$

(ii) $\delta_{21}<\delta_{12}$

(iii) $\delta_{21}>\delta_{12}$

We assume that $\mu_{1}=\mu_{2}=\mu$ in all cases. This assumption is understandable, since $\mu_{1}$ and $\mu_{2}$ are natural birth/death rate in SEC 1 and SEC 2, respectively.

Case 1. This is a situation where susceptible humans move across SECs at an equal rate (i.e., $\delta_{12}=\delta_{21}$ ).

In this case, the DFE becomes

$$
\left(S_{1}^{*}, I_{1}^{*}, S_{2}^{*}, I_{2}^{*}, P^{*}, Q^{*}\right)=\left(\frac{N}{2}, 0, \frac{N}{2}, 0, Z, 0\right),
$$

and $\mathscr{R}_{h 1}$ and $\mathscr{R}_{h 2}$ becomes

$$
\mathscr{R}_{h 1}=\frac{\beta_{1} N}{2\left(\mu_{1}+\rho_{1}\right)}, \quad \mathscr{R}_{h 2}=\frac{m \beta_{1} N}{2\left(\mu_{2}+a \rho_{1}\right)} .
$$

Since $m<1$ and $a>1$ then $\mathscr{R}_{h 2}<\mathscr{R}_{h 1}$. This means that $\mathscr{R}_{h}=\mathscr{R}_{h 1}$, since $\mathscr{R}_{h}$ is the greatest eigenvalue with the assumption that there is uniform migration or movement rates. We can infer from this that the outbreak of the disease will be driven by the lower SEC. Therefore, the control intervention strategy should be targeted more at the lower SEC for effective reduction of the spread of Lassa fever disease.

Case 2. The case considered here, is when there is more movement of susceptible humans from the higher SEC to the lower SEC (i.e., $\left.\delta_{12}<\delta_{21}\right)$. Hence, $\mathscr{R}_{h 1}$ and $\mathscr{R}_{h 2}$ can be written as $\mathscr{R}_{h 1}=\beta_{1} \delta_{21} N /\left(\mu_{1}+\rho_{1}\right)\left(\delta_{21}+\delta_{12}\right)$, and $\mathscr{R}_{h 2}=m$ $\beta_{2} \delta_{12} N /\left(\mu_{2}+a \rho_{2}\right)\left(\delta_{21}+\delta_{12}\right)$. And since $m<1, a>1$ and also $\delta_{12}<\delta_{21}$, it is easy to see again that $R_{h 2}<R_{h 1}$. This result is seen to be in agreement with the result derived from the first case, where there is equal movement from one SEC to the other. This implies that uniform movement between SECs will give the same result as when more individuals move from higher SEC to lower SEC.

Case 3. This is the case where there is more movement of susceptible humans from the lower SEC to the higher SEC. To determine the dominant SEC, we take two extreme cases: when the difference between SEC 1 and SEC 2 is considerably small (i.e., $m \rightarrow 1, a \rightarrow 1$ ) and when the difference between one SEC and the other is large (i.e., $m \rightarrow 0, a \rightarrow \infty$ ). By taking the limits of the basic reproduction numbers $\left(\mathscr{R}_{h 2}, \mathscr{R}_{h 1}\right)$ and comparing the values obtained from the limits, we obtained

$$
\begin{aligned}
& \mathscr{R}_{h 1}<\mathscr{R}_{h 2} \quad \text { as } \quad m \rightarrow 1, a \rightarrow 1, \\
& \mathscr{R}_{h 2}<\mathscr{R}_{h 1} \quad \text { as } \quad m \rightarrow 0, a \rightarrow \infty .
\end{aligned}
$$

From the result above, we can infer that if the movement rate is greater into the SEC 2, then this SEC 2 (higher SEC) will drive the Lassa fever outbreak, provided there is a consid- erable small difference between the two socioeconomic classes.

3.4. Outbreak Growth Rate of the 2-SEC Model. If $\mathscr{R}_{0}>1$, it will make the DFE (5) to be unstable thereby leading to outbreak of Lassa fever disease. It is necessary to find out the rate of growth of Lassa fever outbreak in the population. So, we will consider the outbreak growth rate when the basic reproduction number is greater than unity to have a good understanding of the dynamics of the disease at this stage and as well inform the public health for proper management of the outbreak. The outbreak growth rate is determined as the positive (dominant) eigenvalue of the Jacobian evaluated at the DFE [32]. The eigenvalues of the Jacobian of model (4) evaluated at the DFE (5) are

$$
\begin{aligned}
& \lambda_{1}=-\left(\mu_{1}+\delta_{12}\right), \\
& \lambda_{2}=-\left(\mu_{2}+\delta_{21}\right), \\
& \lambda_{3}=\frac{\left(b_{22}+b_{44}\right)-\sqrt{\left(b_{22}+b_{44}\right)^{2}+4\left(l_{21} l_{12}-b_{22} b_{44}\right)}}{2}, \\
& \lambda_{4}=\frac{\left(b_{22}+b_{44}\right)+\sqrt{\left(b_{22}+b_{44}\right)^{2}+4\left(l_{21} l_{12}-b_{22} b_{44}\right)}}{2}, \\
& \lambda_{5}=-\xi, \\
& \lambda_{6}=\xi\left(\mathscr{R}_{r}-1\right),
\end{aligned}
$$

where $b_{22}=\left(\mu_{1}+\rho_{1}+l_{12}\right)\left(\mathscr{R}_{11}-1\right), \quad b_{44}=\left(\mu_{2}+\rho_{2}+l_{21}\right)($ $\left.\mathscr{R}_{22}-1\right), \quad \mathscr{R}_{11}=\left(\beta_{1} S_{1}^{0}\right) /\left(\mu_{1}+\rho_{1}+l_{12}\right), \quad \mathscr{R}_{22}=\left(\beta_{2} S_{2}^{0}\right) /\left(\mu_{2}+\right.$ $\left.\rho_{2}+l_{21}\right)$. The two possible dominant positive eigenvalue (which the maximum one is the outbreak growth rate) are $\lambda_{4}$ and $\lambda_{6}$. Clearly, $\lambda_{6}>0$ if $\mathscr{R}_{r}>1$. To show that $\lambda_{4}>0$, observe that $\lambda_{4}$ will be positive if $\mathscr{R}_{11} \geq 1, \mathscr{R}_{22} \geq 1$ and $\left(b_{22}+b_{44}\right)^{2}+4\left(l_{21} l_{12}-b_{22} b_{44}\right) \geq 0$. To verify this, we consider the following cases. If $\mathscr{R}_{11}=1$ and $\mathscr{R}_{22}=1$, then $\lambda_{4}=$ $\sqrt{l_{12} l_{21}}$ which is positive. Also, if $l_{12}=0$ or $l_{21}=0$, then $\lambda_{4}$ $=b_{22}$ or $b_{44}$ which are both positive, provided $\mathscr{R}_{11}>1$ and $\mathscr{R}_{22}>1$. For various parameter range considered, we obtain $\lambda_{4}>0$. Thus, the outbreak growth rate of the homogeneous model (4) is

$$
\lambda^{+}=\max \left\{\lambda_{4}, \lambda_{6}\right\}
$$

provided $\mathscr{R}_{r}>1, \mathscr{R}_{11} \geq 1, \mathscr{R}_{22} \geq 1$ and $\left(b_{22}+b_{44}\right)^{2}+4\left(l_{21}\right.$ $\left.l_{12}-b_{22} b_{44}\right) \geq 0$. This shows that at the endemic stage of the outbreak when the basic reproduction number is greater than unity, the rate at which the outbreak grows depends completely on either rats or humans factors.

Furthermore, from equation (20), we can see that if $\mathscr{R}_{r}$ $<1, \mathscr{R}_{11}<1$, and $\mathscr{R}_{22}<1$, all the eigenvalues will have a negative real part. This confirms that the DFE is locally stable when $\mathscr{R}_{r}<1, \mathscr{R}_{11}<1$, and $\mathscr{R}_{22}<1$. Epidemiologically, this implies that Lassa fever can be eliminated from the human population if the size of the initial population of the infected 
human and rats lies in the neighbourhood of the DFE, provided the basic reproduction number is less than unity.

3.5. Model Simulations. In this section, we present numerical simulations of our model to support our analytical results. In particular, we investigate numerically the effects of migration of humans across SECs on the dynamics of the disease. In our analytical results in the previous sections, we assumed that $l_{12}=l_{21}=0$. However, in the numerical simulation here, we will drop this assumption and consider realistic parameter values for $l_{12}$ and $l_{21}$. The parameter values used for the numerical simulation are presented in Table 3. Based on these parameter values, we present the following numerical simulations.

Figure 1 illustrates the case where there are no movements across SECs. We discovered from the figure that SEC 1 drives the outbreak in this case. This agrees with our analytical result of Case 1 . We also notice from the figure that there is a huge difference on the dynamics of infected population for the two SECs. Thus, whenever Lassa fever outbreak occurs in a society where there is no movement across the socioeconomic classes in the population, the lower socioeconomic class should be the target of control measures for maximum reduction on the spread of the disease.

From Figure 2, we discovered that when there is an equal rate of movement across SECs, the SEC 1 dominates and drive the outbreak over SEC 2. This also agrees with our analytic result in Case 1. For this case, we notice from the figure a small difference on the infected population dynamics for the two socioeconomic classes. Therefore, whenever Lassa fever outbreak occurs in a society where there is equal movement across the socioeconomic classes, the two socioeconomic classes should be the target of control measures in other to reduce the spread of the disease.

From Figure 3, we discovered also that when there is less movement from the lower SEC to the higher SEC, the lower SEC still drives the outbreak. This also agrees with our analytic result in Case 2. Based on these results, we recommend that whenever Lassa fever outbreak occurs in a society where movement rate into the lower socioeconomic class is greater, the lower socioeconomic class should be the target of control measures for effective reduction in the spread of the disease.

From Figure 4, we discovered that when there is more movement from lower SEC to higher SEC, the higher SEC drives the outbreak. The infection growth rate in SEC 1 is still high but not compared to when the movement from SEC 2 to SEC 1 is higher. Based on these results, we recommend that whenever Lassa fever outbreak occurs in a society where movement rate into the higher socioeconomic class is greater, the higher socioeconomic class should be the target of control measures for effective reduction of the spread of the disease. Note that all these agree with our earlier analytical results. Since consideration of SEC gives a more realistic view of the dynamics of Lassa fever disease in a multiple socioeconomic society, the results of our study can be used in advising the policy makers for effective management and control of Lassa fever.
TABLE 3: Parameter values used for the numerical simulations.

\begin{tabular}{lcc}
\hline Symbol of the parameters & Parameter values & Source \\
\hline$\mu_{j}$ & 0.0000548 & {$[9]$} \\
$\beta$ & 0.00002 & {$[10]$} \\
$\beta_{1}$ & $1.5 \beta$ & Estimated \\
$\beta_{2}$ & $0.5 \beta$ & Estimated \\
$\alpha$ & 0.00001 & {$[10]$} \\
$\alpha_{1}$ & $1.5 \alpha$ & Estimated \\
$\alpha$ & $0.5 \alpha$ & Estimated \\
$\rho$ & 0.0476 & {$[9]$} \\
$\rho_{1}$ & $0.5 \rho$ & Estimated \\
$\rho_{2}$ & $1.5 \rho$ & Estimated \\
$\xi$ & 0.2 & {$[9]$} \\
$\varphi$ & 0.002 & {$[11]$} \\
$\delta 12$ & $0.2-0.4$ & {$[27]$} \\
$\delta 21$ & $0.2-0.4$ & {$[27]$} \\
$l 12$ & $\delta 12$ & {$[27]$} \\
$l 21$ & $\delta 21$ & {$[27]$} \\
\hline
\end{tabular}

Note: $\beta, \alpha$, and $\rho$ represent human to human contact rate, human to rats contact rate, and recovery rate of human, respectively, in a homogeneous population (i.e., population where SECs are not considered). We considered the above data for a homogeneous population due to limited access to data on SECs.

3.6. Sensitivity Analysis. To understand the importance of different model parameters responsible for disease transmission as well as its prevalence, it is important to carry out a sensitivity analysis of the basic reproduction number. According to [31], the initial disease transmission has a direct relation with the basic reproduction number. We compute the sensitivity indices of the basic reproduction numbers $\left(R_{h 1}, R_{h 2}\right)$ of equation (4) with respect to the movement rates $\delta_{12}$ and $\delta_{21}$. The indices calculated will give insight into the relevance of each parameter to the prevalence of the disease. The parameter with greater index (in magnitude) should be taken into consideration (with higher priority) while defining control strategy. The normalized forward sensitivity index of a variable, $u$, that depends differentiable on a parameter, $\%$, is defined as follows:

$$
Y_{\rho}^{u}=\frac{\partial u}{\partial \rho} \times \frac{\rho}{u}
$$

When $Y_{\rho}^{u}>0$, we say that \% increases the value of $u$ as its value increases, while if $Y_{\rho}^{u}<0$, we say that $\%$ decreases the value of $u$ as its value increases.

From (21) above, it is obvious that we have an explicit formula for calculating the sensitivity indices of the basic reproduction numbers $\left(\mathscr{R}_{h 1}, \mathscr{R}_{h 2}\right)$. Therefore, we proceed to determine the sensitivity indices of these reproduction numbers $\left(\mathscr{R}_{h 1}, \mathscr{R}_{h 2}\right)$ with respect to $\delta_{12}$ and $\delta_{21}$. By taking the values of the movement rate as $\delta_{12}=0.35$ and $\delta_{21}=$ 0.25 , we obtain the following: 


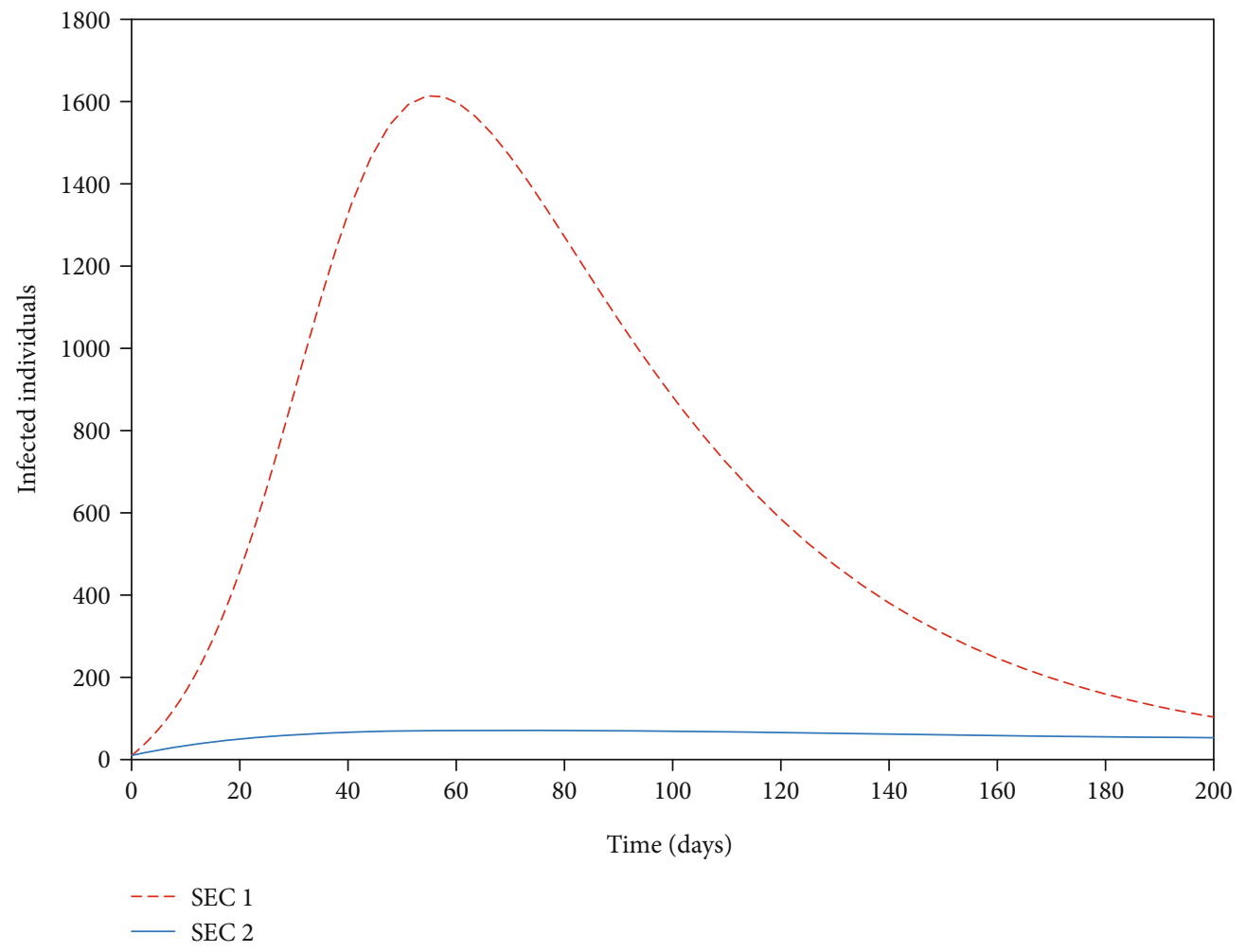

FIGURE 1: Figure illustrating the possible dynamics of SEC 1 and SEC 2 using model (19) for $\delta_{12}=\delta_{21}=0$.

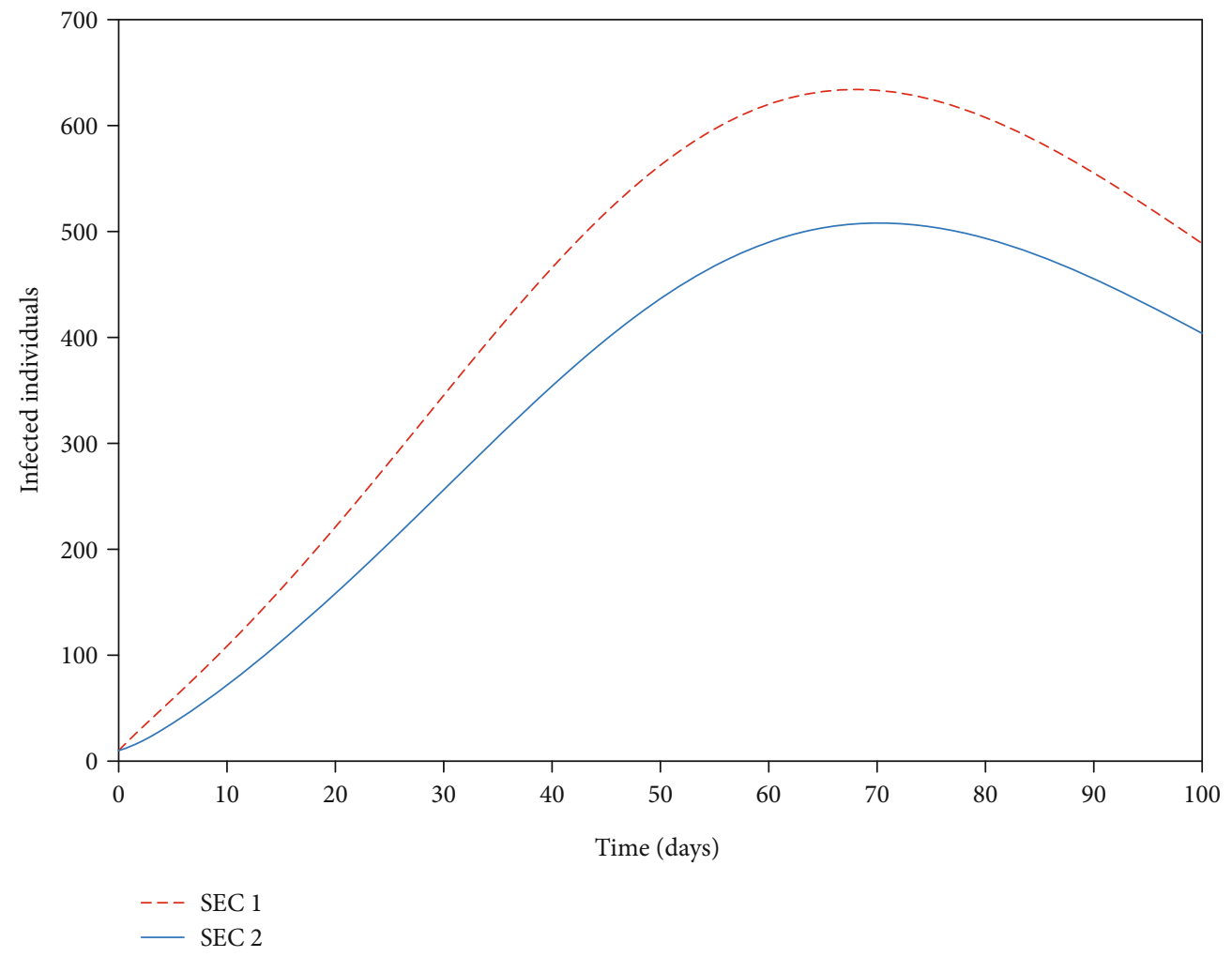

FIgURE 2: Figure illustrating the possible dynamics of SEC 1 and SEC 2 using model (19) for $\delta_{12}=\delta_{21}=0.2$. 


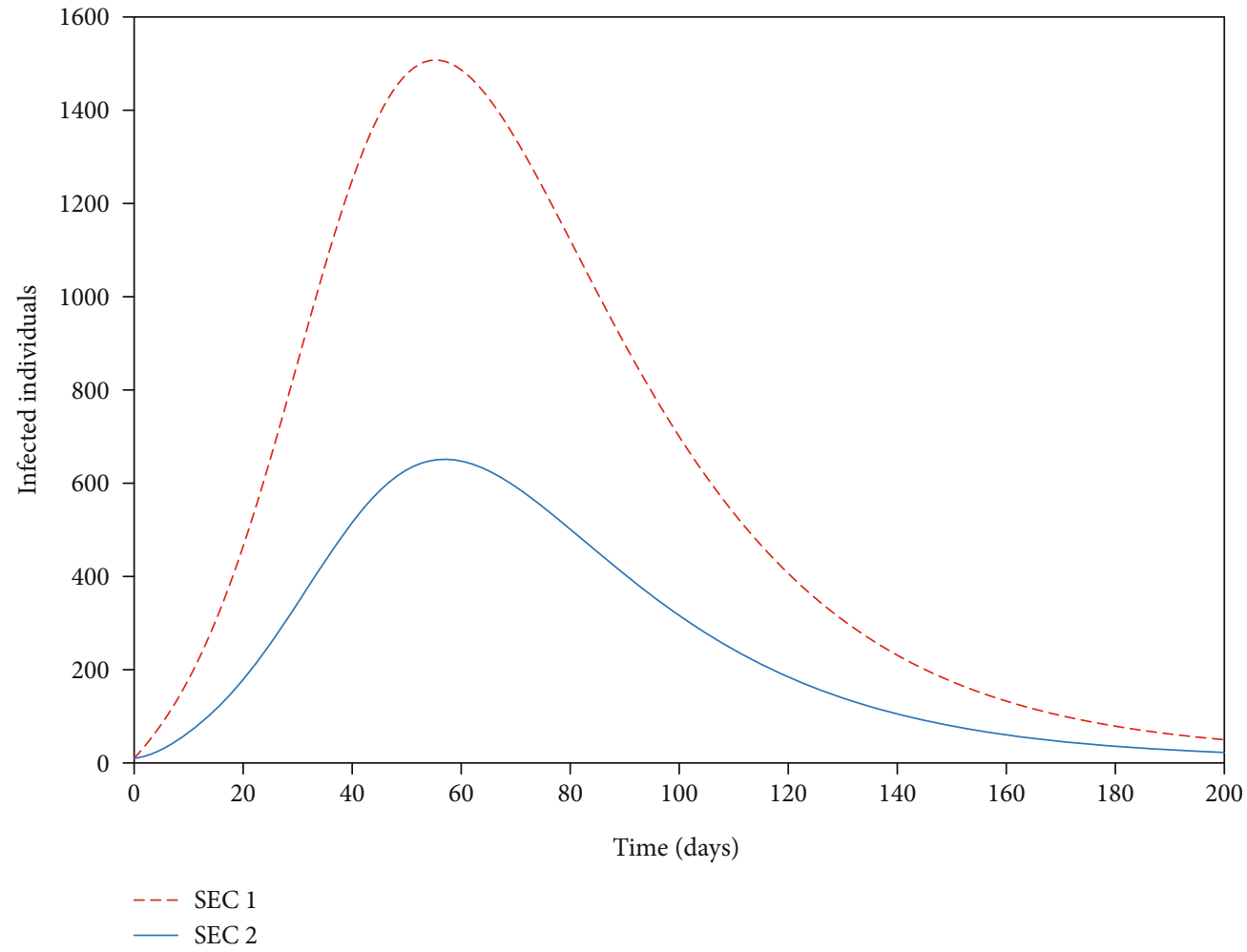

FIgURE 3: Figure illustrating the possible dynamics of SEC 1 and SEC 2 using model (19) for $\delta_{12}=0.2$ and $\delta_{21}=0.4$.

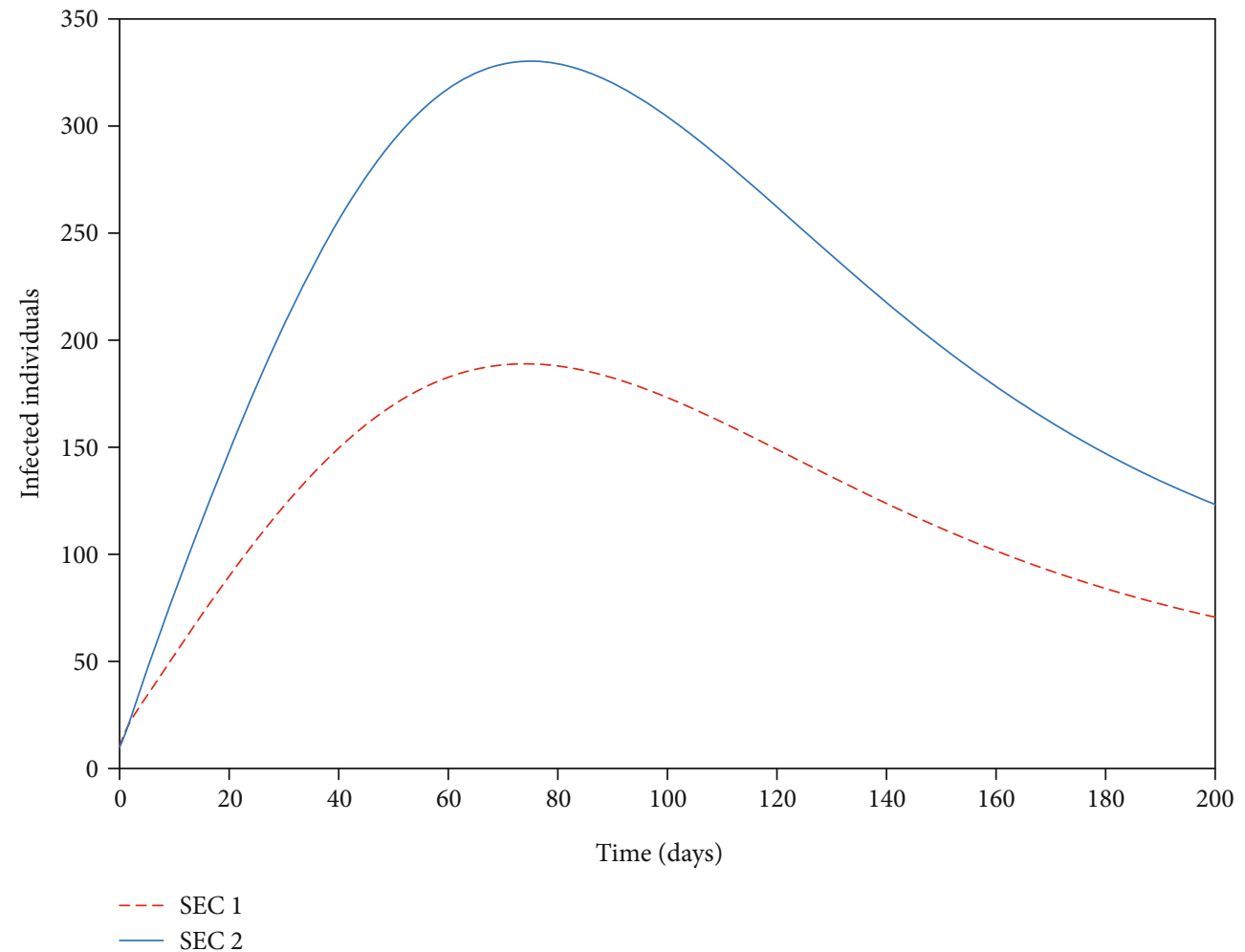

FIgURE 4: Figure illustrating the possible dynamics of SEC 1 and SEC 2 using model (19) for $\delta_{12}=0.4$ and $\delta_{21}=0.2$. 
$Y_{\delta_{12}}^{\mathscr{R}_{h 1}}=-0.5833, Y_{\delta_{21}}^{\mathscr{R}_{h 1}}=0.5833, Y_{\delta_{12}}^{\mathscr{R}_{h 2}}=0.4167, Y_{\delta_{21}}^{\mathscr{R}_{h 2}}=-0.4167$

From the above results, it is very clear that movement rates $\delta_{12}$ and $\delta_{21}$ have a significant impact on the basic reproduction number and consequently the disease dynamics. Thus, they are among the parameters that should be taken into consideration while defining control strategy. Also, we can see that the sensitivity index of $\mathscr{R}_{h 1}$ with respect to $\delta_{12}$ and $\delta_{21}$ has equal magnitude but opposite signs. Thus, $\delta_{12}$ and $\delta_{21}$ has equal but opposite effects on the reproduction number $\mathscr{R}_{h 1}$. Epidemiologically, this implies that an increase in $\delta_{12}$ decreases $\mathscr{R}_{h 1}$ while an increase in $\delta_{21}$ increases $\mathscr{R}_{h 1}$. Similarly, the sensitivity index of $\delta_{12}$ with respect to $\delta_{12}$ and $\delta_{21}$ has equal magnitude but opposite effects. Thus, the rate at which individuals migrate from SEC 1 decreases $\mathscr{R}_{h 1}$, while the rate at which humans migrate into SEC 1 increases $R_{h 1}$. Similarly, the rate at which individuals migrate from SEC 2 decreases $\mathscr{R}_{h 2}$, while the rate at which humans move into SEC 2 increases $\mathscr{R}_{h 2}$.

\section{The $n$-Socioeconomic Class Model}

In this section, we extend some of the results we have in the 2-SEC model (4) to the general $n$-socioeconomic class model (1).

4.1. Disease Free Equilibrium for the n-SEC Model. The unique DFE of the $n$-SEC model (1) is given by

$$
E^{0}=\left(S_{1}^{0}, I_{1}^{0}, S_{2}^{0}, I_{2}^{0}, \cdots, S_{n}^{0}, I_{n}^{0}, P^{0}, Q^{0}\right)
$$

where $S_{1}^{0}=N /\left(1+\left(\delta_{12} / \delta_{21}\right)+\left(\delta_{12} / \delta_{21}\right)\left(\delta_{23} / \delta_{32}\right)+\left(\delta_{12} / \delta_{21}\right)\right.$ $\left(\delta_{23} / \delta_{32}\right)\left(\delta_{34} / \delta_{43}\right)+\cdots+\left(\delta_{12} / \delta_{21}\right)\left(\delta_{23} / \delta_{32}\right)\left(\delta_{34} / \delta_{43}\right)+\cdots+(\delta$ $\left.\left.(n-1) n=\delta_{n(n-1)}\right)\right), \quad S_{2}^{0}=\left(\delta_{12} / \delta_{21}\right) S_{1}^{0}, \quad S_{3}^{0}=\left(\delta_{12} / \delta_{21}\right)\left(\delta_{23} / \delta_{32}\right) S_{1}^{0}$, $S_{4}^{0}=\left(\delta_{12} / \delta_{21}\right)\left(\delta_{23} / \delta_{32}\right)\left(\delta_{34} / \delta_{43}\right) S_{1}^{0}, \cdots S_{n}^{0}=\left(\delta_{12} / \delta_{21}\right)\left(\delta_{23} / \delta_{32}\right)$ $\left(\delta_{34} / \delta_{43}\right) \cdots\left(\delta_{n-1 n} / \delta_{n n-1}\right) S_{1}^{0} \quad \cdot \quad\left(I_{1}^{0}, I_{2}^{0}, \cdots, I_{n}^{0}\right)=(0,0, \cdots, 0)$, and $\left(P^{0}, Q^{0}\right)=(Z, 0)$.

4.2. The Basic Reproduction Number for $n-S E C$ Model. From the expression for the DFE of the $n$-SEC model, if we take into consideration a case similar to the 2-SEC model, assuming no movement of infected humans from one SEC to another, then the basic reproduction number of the model is derived and given as

$$
\mathscr{R}^{*}=\max \left\{\mathscr{R}_{h j}, \mathscr{R}_{r}\right\} j=1,2,3, \cdots, n,
$$

where

$$
\mathscr{R}_{h j}=\frac{m^{j-1} \beta_{1} N}{\left(\mu_{j}+a^{j-1} \rho_{1}\right)} \frac{\delta_{12}}{\delta_{21}} \frac{\delta_{23}}{\delta_{32}} \frac{\delta_{34}}{\delta_{43}} \cdots \frac{\delta_{j-1 j}}{\delta_{j j-1}} S_{1}^{0} .
$$

Note that the threshold $R_{h j}$ is the basic reproduction number of the human compartment of SEC $j$ of model (1). To determine the SEC that is driving the outbreaks in the human population of the general $n$-SEC model, we take into consideration the same cases as in 2-SEC as follows:

Case i. $\delta_{j k}=\delta_{k j} \cdot \forall j, k$.

Considering this assumption, the DFE (23) becomes

$$
\left(S_{1}^{0}, I_{1}^{0}, S_{2}^{0}, I_{2}^{0}, \cdots, S_{n}^{0}, I_{n}^{0}\right)=\left(\frac{N}{n}, 0, \frac{N}{n}, 0, \cdots, \frac{N}{n}, 0\right),
$$

while the basic reproduction number (25) becomes

$$
\mathscr{R}_{h j}=\frac{m^{j-1} \beta_{1} N}{n\left(\mu_{j}+a^{j-1} \rho_{1}\right)} .
$$

We can show easily that

$$
\mathscr{R}_{h j+1}<\mathscr{R}_{h j}, \quad j=1,2,3, \cdots, n .
$$

The result of equation (28) shows that when there is equal movement from one SEC to another, the lower SEC dominates and hence will drive the outbreak. This notwithstanding, control strategies should be targeted at all SECs with $\mathscr{R}_{h j}>1$ but with more emphasis on the lower SEC.

Case ii. $\delta_{j k}<\delta_{k j}$ for $j<k$, this is a case where more humans migrate from higher to lower SECs. This case gives the same result as in (28).

Case iii. $\delta_{j k}>\delta_{k j}$ for $j<k$. This is a case where more individuals migrate from lower to higher SECs.

For this case, the inequality $\mathscr{R}_{h j}<\mathscr{R}_{h j+1}$, as $m \rightarrow 1, a \rightarrow 1$ holds.

These results show that there is a possibility of any of the SECs driving the outbreak if there are more movements of humans into that SEC, and the population size gets too big. The movement rates that will necessitate this action changes with respect to other model parameters. When movement into SECs that are higher becomes predominant, then control strategies should be channelled to these higher SECs, since they will eventually drive the outbreak of the disease.

\section{Discussion}

Socioeconomic status of humans in a given population is known to affect the transmission dynamics of any infectious disease in a community. In this study, we formulated an $n$ - patch Lassa fever disease model to enable us determine the impact of socioeconomic status in the transmission dynamics of Lassa fever disease in a population. Initial insight into the model is gained by considering particular case of two socioeconomic classes.

In analysing the model, we took a special case of two socioeconomic classes in a community and determined and analysed some basic mathematical and epidemiological features of the model. Some of the epidemiological features of 
the model determined include the basic reproduction number, the type reproduction number, and outbreak growth rate. Dynamical system analysis of these epidemiological features reveals some transmission dynamics of Lassa fever in a two socioeconomic population. For instance, we discovered from our analyses that complete eradication of Lassa fever is possible when SEC is considered, as long as the value of the basic reproduction number is kept at a value less than unity. On the other hand, there will be an outbreak if the reproduction number is greater than unity.

Next, we investigated the differences in the dynamics of the two socioeconomic classes based on the rates across the socioeconomic classes. For these investigations, we considered three special cases. In the first case, we considered the situation where there is equal migration rates. From our analyses, we saw that the SEC 1 will have more number of infections when compared with the SEC 2. This shows that SEC 1 will be the dominant and consequently drive the outbreak of Lassa fever in the population. Therefore, to effectively control the outbreak of Lassa fever in a community, the SEC 1 will be highly considered in providing effective disease control measures.

The second case, we considered is a situation where more humans migrate from SEC 2 to SEC 1. In this case, we discovered that the number of infected individuals in SEC 1 will still dominate that of SEC 2. For the third case, we considered more migrations from SEC 1 to SEC 2 and obtained a different result which is that the number of infections generated in SEC 2 started dominating. This shows that any SEC can drive the outbreak when it has more population of infected and susceptible humans. So, we recommend that control intervention measures should be concentrated more on any SEC that has a greater migration rate coming into it, as it can be seen that the migration rate plays a vital role in determining the SEC that drives the outbreak of Lassa fever disease. Since, considering SEC is more realistic, it implies that considering socioeconomic classes should be encouraged for a better understanding of the dynamics of Lassa fever and consequent development of better control intervention strategies.

Sensitivity analysis of our model was carried out to determine the impact of movement rate across the socioeconomic classes to initial disease transmission. The results of the analysis revealed that the movement rate have a significant impact on initial disease transmission. Also, the sensitivity analysis results also show that the rate of immigration and emigration into any SEC has opposite impact on the initial disease transmission and prevalence. This suggests that the movement rate should be put into consideration in defining control measures to reduce the prevalence of the disease in the entire population.

In conclusion, we extended the facts established in the results of the 2-SEC model to a general $n$-SEC model. We discovered that the dynamical behaviour of our model agrees with real-life expectation as far as Lassa fever disease is concerned. Therefore, this model can be used in studying the dynamics of Lassa fever, so as to predict the future Lassa fever outbreak in communities where the disease is endemic. The findings from our study have given us a reason to conclude that the socioeconomic status of humans has a significant impact on Lassa fever transmission dynamics. Thus, socioeconomic classes of humans should be put into considerations in recommending effective control intervention measures to be used for total eradication of Lassa fever in communities where the disease is endemic.

Even though our model has been used successfully to study the dynamics of Lassa fever, it is important to know that this model has limitations. We assumed constant birth rate and death rate for all socioeconomic classes resulting in constant population. However, it is expected that the higher SEC should have a higher life expectancy than the lower SEC. Another limitation of this study is that our model has not been validated using real data. This is due to limited access to data. All these limitations will be taken into consideration in our future study.

\section{Data Availability}

The data supporting this deterministic model are from previously published articles and they have been duly cited in this paper.

\section{Conflicts of Interest}

The authors declare that there are no conflicts of interest regarding the publication of this paper.

\section{References}

[1] M. L. Cohen, "Changing patterns of infectious disease," Nature, vol. 406, no. 6797, pp. 762-767, 2000.

[2] W. H. Fleming and R. W. Rishel, "Deterministic and stochastic optimal control," Bulletin American Mathematical Society, vol. 82, pp. 869-870, 1976.

[3] World Health Organisation (WHO), "Lassa feverwebsite," 2018.

[4] World Health Oragnization (WHO)(february, 2020), https:// www.who.int/csr/don/20-february-2020-lassa-fevernigeria/ en/.

[5] The American Heritage @ New Dictionary of Cultural Literacy, Third edition, , 2013http://dictionary.reference.com/browse/ socioeconomicstatus.

[6] P. J. Hotez, A. Fenwick, L. Savioli, and D. H. Molyneux, "Rescuing the bottom billion through control of neglected tropical diseases," The Lancet, vol. 373, no. 9674, pp. 1570-1575, 2009.

[7] H. U. Okoroiwu, F. Lopez-Munoz, and F. J. Povedano-Montero, "Bibliometric analysis of global Lassa fever research (1970-2017): a 47-year study," BMC Infectious Diseases, vol. 18, no. 1, p. 639, 2018.

[8] M. Bawa, S. Abdulraham, and O. R. Jimoh, "Stability analysis of the disease-free equilibrium state of Lassa fever disease," International Journal Science Mathematics Education, vol. 9, pp. 115-123, 2013.

[9] E. A. Bakare, E. B. Are, O. E. Abolarin, S. A. Osanyinlusi, B. Ngwu, and O. N. Ubaka, "Mathematical Modelling and Analysis of Transmission Dynamics of Lassa Fever," Journal of Applied Mathematics, vol. 2020, 18 pages, 2020.

[10] O. S. Obabiyi and A. A. Onifade, "Mathematical model for Lassa fever transmission dynamics with variable human and 
reservoir population," International Journal Differential Equation Application, vol. 16, 2017.

[11] M. B. Abdullahi, U. C. Doko, and M. Mamuda, "Sensitivity analysis in a Lassa fever deterministic mathematical model," in , Article ID 050050AIP Conference Proceedings, vol. 1660, Penang, Malaysia, 2015.

[12] S. C. Nwasuka, I. E. Nwachukwu, and P. C. Nwachukwu, "Mathematical Model of the Transmission Dynamics of Lassa Fever with Separation of Infected Individual and Treatment as Control Measures," Journal Advances in Mathematics and Computer Science, vol. 32, pp. 1-15, 2019.

[13] G. L. Iacono, A. A. Cunningham, E. Fichet-Calvet et al., "Using modelling to disentangle the relative contributions of zoonotic and anthroponotic transmission: the case of Lassa fever," PLoS Neglected Tropical Diseases, vol. 9, no. 1, p. e3398, 2015.

[14] I. S. Onah, O. C. Collins, P. G. U. Madueme, and G. C. E. Mbah, "Dynamical System Analysis and Optimal Control Measures of Lassa Fever Disease Model," International Journal of Mathematics and Mathematical Sciences, vol. 2020, 18 pages, 2020.

[15] S. S. Musa, S. Zhao, D. Gao, Q. Lin, G. Chowell, and D. He, "Mechanistic Modelling of the Largescale Lassa Fever Epidemics in Nigeria from 2016 to 2019," Journal Theoretical Biology, vol. 493, p. 110209, 2020.

[16] J. Mariën, B. Borremans, F. Kourouma et al., "Evaluation of rodent control to fight Lassa fever based on field data and mathematical modelling," Emerging Microbes and Infections, vol. 8, no. 1, pp. 640-649, 2019.

[17] A. R. Akhmetzhanov, Y. Asai, and H. Nishiura, "Quantifying the seasonal drivers of transmission for Lassa fever in Nigeria," Philosophical Transactions of the Royal Society B, vol. 374, no. 1775, 2019.

[18] S. Zhao, S. S. Musa, H. Fu, D. He, and J. Qin, "Large-scale Lassa fever outbreaks in Nigeria: quantifying the association between disease reproduction number and local rainfall," Epidemiology and Infection, vol. 148, 2020.

[19] E. A. Ilori, Y. Furuse, O. B. Ipadeola et al., "Epidemiologic and Clinical Features of Lassa Fever Outbreak in Nigeria, January 1 -May 6, 2018," Emerging Infectious Disease, vol. 25, pp. 10661074, 2019.

[20] L. Roberts, "Nigeria hit by unprecedented Lassa fever outbreak," Science, vol. 359, no. 6381, pp. 1201-1202, 2018.

[21] N. A. Ajayi, C. G. Nwigwe, B. N. Azuogu et al., "Containing a Lassa fever epidemic in a resource-limited setting: outbreak description and lessons learned from Abakaliki, Nigeria (January-March 2012)," International Journal Infectious Disease, vol. 17, pp. 1011-1016, 2012.

[22] B. M. Warner, D. Safronetz, and D. R. Stein, "Current research for a vaccine against Lassa hem-orrhagic fever virus," Drug Design, Development and Therapy, vol. 12, pp. 2519-2527, 2018.

[23] I. S. Lukashevich, "The search for animal models for Lassa fever vaccine development," Expert Review of Vaccines, vol. 12, pp. 71-86, 2014.

[24] I. S. Lukashevich and P. Pushko, "Vaccine platforms to control Lassa fever," Expert Review of Vaccines, vol. 15, no. 9, pp. 1135-1150, 2016.

[25] L. M. Branco, J. N. Grove, F. J. Geske et al., "Lassa virus-like particles displaying all major immunological determinants as a vaccine candidate for Lassa hemorrhagic fever," Virology Journal, vol. 7, no. 1, p. 279, 2010.
[26] P. Van den Driessche and J. Watmough, "Reproduction numbers and sub-threshold endemic equilibria for compartmental models of disease transmission," Mathematical Biosciences, vol. 180, no. 1-2, pp. 29-48, 2002.

[27] O. C. Collins, S. L. Robertson, and K. S. Govinder, "Analysis of a waterborne disease model with socioeconomic classes," Mathematical Biosciences, vol. 269, pp. 86-93, 2015.

[28] J. A. P. Heesterbeek and M. G. Roberts, "The typereproduction number $\mathrm{T}$ in models for infectious disease control," Mathematical Biosciences, vol. 206, no. 1, pp. 3-10, 2007.

[29] M. G. Roberts and J. A. P. Heesterbeek, "A new method for estimating the effort required to control an infectious disease," Proceedings of the Royal Society of London Series B: Biological Sciences, vol. 270, no. 1522, pp. 1359-1364, 2003.

[30] World Health Oragnization (WHO)July, 2020, https://www .who.int/news-room/fact-sheets/detail/lassa-fever.

[31] O. Diekmann, J. A. P. Heesterbeek, and M. G. Roberts, "The construction of next-generation matrices for compartmental epidemic models," Journal Royal Society Interface, vol. 7, pp. 873-885, 2010.

[32] J. M. Tchuenche, S. A. Khamis, F. B. Agusto, and S. C. Mpeshe, "Optimal control and sensitivity analysis of an influenza model with treatment and vaccination," Acta Biotheoretica, vol. 59, no. 1, pp. 1-28, 2011. 